\title{
THE RECONSTRUCTION OF CLUSTER MASS PROFILES FROM IMAGE DISTORTIONS
}

\author{
PETER SCHNEIDER \\ Max-Planck-Institut für Astrophysik \\ Postfach 1523, D-85740 Garching, Germany
}

\begin{abstract}
I will review the basic principles and some of the most recent developments of parameter-free reconstructions of cluster mass profiles from the distortions of background galaxy images.
\end{abstract}

\section{Introduction}

The discovery of cluster lensing in the form of giant luminous arcs was immediately recognized as a new tool for determining the mass in the inner part of the lensing clusters. Less dramatic image distortions as seen in arclets (Fort et al. 1988) and in the coherent image alignments (Tyson et al. 1990) have increased the angular extent over which mass estimates can be obtained - out to about $1 \mathrm{Mpc}$ in some cases (see, e.g., Bonnet et al. 1994). A detailed account of the observation, theory and modeling of arc clusters is given in Fort \& Mellier (1994). In their pioneering paper, Kaiser \& Squires (1993; hereafter KS) have shown that the observed image ellipticities can be directly translated into an estimate for the surface mass distribution of the cluster lens, without the need for fitting parametrized mass models. The underlying idea is that the image distortions are caused by the tidal gravitational field, which in turn is directly related to the mass distribution. The KS inversion method, described in the next section, has already been applied to a number of clusters (Fahlman et al. 1995; Smail et al. 1995), and further applications can be found elsewhere in these proceedings (see contributions by N. Kaiser, C. Seitz); at least in one case a surprisingly large mass-to-light ratio was found (Fahlman et al. 1995). The faint level to which background galaxies can now be imaged, with the corresponding high number density of objects, together with the imaging capability of 
the HST, the improvements in ground-based imaging, the increase of the field of view, and the development of improved inversion techniques, render the mass inversion from image distortion a unique tool for studying the mass distribution in clusters of galaxies. Here I shall review some of the latest developments of techniques for the reconstruction of the cluster mass distribution.

\section{The "classical" Kaiser \& Squires method}

The shear (or tidal field) is described by a traceless symmetric $2 \times 2$ tensor, or, equivalently, by a two-component quantity, for which we shall use the complex shear $\gamma$, which is related to the dimensionless surface mass density $\kappa$ through $\gamma(\boldsymbol{\theta})=\frac{1}{\pi} \int_{\mathbf{R}^{2}} \mathrm{~d}^{2} \boldsymbol{\theta}^{\prime} \mathcal{D}\left(\boldsymbol{\theta}-\boldsymbol{\theta}^{\prime}\right) \kappa\left(\boldsymbol{\theta}^{\prime}\right)$, with $\mathcal{D}(\mathbf{z})=1 /\left(z_{1}-\mathrm{i} z_{2}\right)^{2}$. The inversion of this convolution-type integral can be easily obtained in Fourier space and yields (KS)

$$
\kappa(\boldsymbol{\theta})=\frac{1}{\pi} \int_{\mathbf{R}^{2}} \mathrm{~d}^{2} \boldsymbol{\theta}^{\prime} \mathcal{R} \mathrm{e}\left[\mathcal{D}^{*}\left(\boldsymbol{\theta}-\boldsymbol{\theta}^{\prime}\right) \gamma\left(\boldsymbol{\theta}^{\prime}\right)\right]+\kappa_{0},
$$

where $\kappa_{0}$ is an arbitrary constant, unconstrained from the $k=0$ mode of the Fourier transform, and an asterisk denotes complex conjugation. Hence, (1) yields a parameter-free estimate of the surface mass distribution, up to an overall additive constant, which, however, can be constrained due to the non-negativity of the mass. Several modifications of this "classical" KS method have been recently suggested in the literature: (a) In general, the shear $\gamma$ is not an observable; however, in the case of weak distortions $(\kappa \ll 1,|\gamma| \ll 1)$ the observable is directly related to $\gamma$ (see Sect.3). The generalization to the strong lensing regime is described briefly in Sect.4. (b) The integral in (1) extends over the 'whole sky', whereas actual data fields are finite. The leads to a bias of (1) at the boundaries of the data field; the generalization to unbiased estimators are described in Sect.5. (c) The integration constant in (1) describes an invariance transformation, which leaves image ellipticities unchanged; this invariance can be broken by invoking magnification effects, which will be briefly outlined in Sect.6.

\section{The observable: distortion}

Unless stated otherwise, we shall, for simplicity, consider in the following the case that the distance ratio $D_{d s} / D_{s}$ is the same for all background sources, which is a good approximation for $z_{d} \lesssim 0.2$. From the tensor $Q_{i j}$ of second brightness moments one defines the complex ellipticity $\chi=\left(Q_{11}-Q_{22}+2 \mathrm{i} Q_{12}\right) / \operatorname{tr} Q$. The locally linearized lens equation yields a transformation between the source and image ellipticity, $\chi^{(s)}=\chi^{(s)}(\chi, g)$, 
where $g=\gamma /(1-\kappa)$ is the 'reduced shear'. With the fundamental assumption, underlying all these reconstruction methods, that the intrinsic orientation of the sources are random, $\left\langle\chi^{(s)}\right\rangle=0$, this translates to an equation determining $g:\left\langle\chi^{(s)}(\chi, g)\right\rangle=0$, where here the angular brackets denote a local average over images, i.e., one introduces a smoothing length. As it turns out, this equation always has two solutions - if $g$ is a solution, so is $1 / g^{*}$. Hence, the observable is the distortion (Kochanek 1990 , MiraldaEscudé 1991, Schneider \& Seitz 1995) $\delta=\frac{2 g}{1+|g|^{2}}=\frac{2 \gamma(1-\kappa)}{(1-\kappa)^{2}+|\gamma|^{2}}$. In the case of weak lensing $(\kappa \ll 1,|\gamma| \ll 1), \gamma \approx \delta / 2 \approx-\langle\chi\rangle / 2$ can be 'observed'. The resulting value for $\delta$ is independent of the intrinsic ellipticity distribution, but the accuracy of the estimate decreases for broader intrinsic distributions. If the intrinsic ellipticity distribution is known, a maximum likelihood estimate yields more precise values for $\delta$, except if the former is a Gaussian.

\section{Non-linear generalization of KS}

The KS inversion (1) can now be written in a form which accounts also for the strong lensing regime, by setting $\gamma=(1-\kappa) g$ :

$$
\kappa(\boldsymbol{\theta})=\frac{1}{\pi} \int_{\mathbf{R}^{2}} \mathrm{~d}^{2} \theta^{\prime}\left[1-\kappa\left(\boldsymbol{\theta}^{\prime}\right)\right] \mathcal{R e}\left[\mathcal{D}^{*}\left(\boldsymbol{\theta}-\boldsymbol{\theta}^{\prime}\right) g\left(\boldsymbol{\theta}^{\prime}\right)\right]+\kappa_{0},
$$

with $g=\delta\left(1-\operatorname{sign}(\operatorname{det} A) \sqrt{1-|\delta|^{2}}\right) /|\delta|^{2}$, and $A$ is the Jacobi matrix of the lens equation. The integral equation (2) for $\kappa$ can be solved iteratively (Seitz \& Schneider 1995a). The resulting mass distribution $\kappa(\theta)$ is then determined up to a global invariance transformation (Schneider \& Seitz 1995)

$$
\kappa(\theta) \rightarrow \lambda \kappa(\theta)+(1-\lambda)
$$

which is the mass sheet degeneracy pointed out by Gorenstein, Falco \& Shapiro (1988) and which leaves the observable $\delta$ invariant. In the linear (weak lensing) case, this reduces to the additive constant in (1). ${ }^{1}$

\section{Unbiased finite-field inversions}

The second modification mentioned in Sect. 2 was to account for the finiteness of the data field $\mathcal{U} \in \mathbb{R}^{2}$. There are two quick fixes to deal with the

\footnotetext{
${ }^{1}$ If the redshift distribution of the sources becomes important, i.e., if $D_{d s} / D_{s}$ is not nearly the same for all sources, this degeneracy is broken, but unless the cluster is nearly critical, an approximate invariance transformation still holds (Seitz \& Schneider 1995c). For critical clusters, the breaking of invariance is stronger and may be used, at least in principle, to fix the mass density uniquely.
} 
$\mathbb{R}^{2}$-integral in (1): one is to set $\gamma=0$ for $\boldsymbol{\theta} \notin \mathcal{U}$ (used in the presently published reconstructions), or, arguably more reasonable, to extrapolate the shear $\gamma$ outside $\mathcal{U}$, as suggested in Seitz \& Schneider (1995a) and quantitatively tested in Bartelmann (1995). The success of these fixes depends on the mass distribution and the CCD field $\mathcal{U}$, as discussed below. The road for an unbiased finite-field inversion was paved by Kaiser (1995) who pointed out that the gradient of $\kappa$ is related to a combination of derivatives of the shear components. He showed that

$$
\nabla \kappa=\mathbf{U} \text { and } \nabla K=\mathbf{u}
$$

where the vector field $\mathbf{U}$ contains first derivatives of the components of the shear $\gamma$, or, alternatively, the gradient of $K=\ln (1-\kappa)$ is related to the reduced shear $g$ and its first derivatives, which is combined to the vector field $\mathbf{u}$. In the linear case, where $\gamma$ is an observable, the first of eq.(4) shows that $\kappa$ can be determined only up to an additive constant, whereas in the general case, $K$ can be determined from the observable $g$ only up to an additive constant, so that we reobtain the mass sheet degeneracy mentioned in the previous section. Either of the two equations (4) can be integrated by path integration - selecting the first for illustration, that becomes $\kappa(\boldsymbol{\theta})=\int_{\boldsymbol{\theta}_{0}}^{\boldsymbol{\theta}} \mathrm{d} \mathbf{l} \cdot \mathbf{U}+\kappa\left(\boldsymbol{\theta}_{0}\right)$, where $\boldsymbol{\theta}_{\mathbf{0}} \in \mathcal{U}$ is a reference point, and $\mathbf{l}$ denotes an integration curve. Averaging over a set $\left\{\boldsymbol{\theta}_{0}\right\}$ of starting points, a generalization reads $\kappa(\boldsymbol{\theta})=\sum_{\left\{\boldsymbol{\theta}_{0}\right\}} w\left(\boldsymbol{\theta}_{0}\right) \int_{\boldsymbol{\theta}_{0}}^{\boldsymbol{\theta}} \mathrm{d} \mathbf{l} \cdot \mathbf{U} / \sum_{\left\{\boldsymbol{\theta}_{0}\right\}} w\left(\boldsymbol{\theta}_{0}\right)+$ const., where the $w\left(\theta_{0}\right)$ are arbitrary weight factors; this yields a set of unbiased finite-field inversions. There remains a huge freedom in selecting the set $\left\{\boldsymbol{\theta}_{0}\right\}$, the weights $w\left(\theta_{0}\right)$ and the curves connecting $\boldsymbol{\theta}_{0}$ and $\boldsymbol{\theta}$, and different choices have been suggested by Schneider (1995) and Kaiser et al. (1995). The reason why different choices are not equivalent is that the vector field $\mathrm{U}$ is obtained from observations and thus is not a gradient field in general if it were a gradient field, even a single line integration would be sufficient. The 'noise' of $\mathbf{U}$ is handled differently by different choices. However, the rotational component of $\mathbf{U}$ is a noise contribution which is readily identified as such, and one can construct a finite-field inversion formula which is designed to filter out this rotational component,

$$
\kappa(\boldsymbol{\theta})=\int_{\mathcal{U}} \mathrm{d}^{2} \boldsymbol{\theta}^{\prime} \mathbf{H}\left(\boldsymbol{\theta}^{\prime} ; \boldsymbol{\theta}\right) \cdot \mathbf{U}\left(\boldsymbol{\theta}^{\prime}\right)+\bar{\kappa}
$$

where the kernel function $\mathbf{H}$ was explicitly constructed in Seitz \& Schneider (1995b); in that paper we have also performed extensive simulations on synthetic data to show that (5) is the best unbiased finite-field method yet 
published. The application of (5) is not more complicated than that of (1), so there is no reason not to apply this unbiased estimator. ${ }^{2}$

\section{Breaking the degeneracy: Magnification effects}

Under the transformation (3), the magnification transforms as $\mu \rightarrow \mu / \lambda^{2}$, i.e. magnification can be used to break the invariance. Two methods have been suggested: Broadhurst et al. (1995; see also contribution by A. Taylor) point out that the local number density $n(>S)$ of background sources with flux $>S$ is changed according to $n(>S)=n_{0}(>S / \mu) / \mu\left(n_{0}\right.$ : unlensed counts). Assuming locally a power law $n_{0} \propto S^{-\alpha}$ one has $n / n_{0}=\mu^{(\alpha-1)}$. $^{3}$ Whereas density fluctuations of the background source population may be a problem for local determination of the magnification, the effect averaged over the whole data field should be able to break the mass sheet degeneracy.

A second method was proposed by Bartelmann \& Narayan (1995, see also contribution by M. Bartelmann), using the fact that the surface brightness $I$ of images is unchanged by lensing. A source of angular size $R_{0}$ thus retains its surface brightness, and its image attains the size $R=\sqrt{\mu} R_{0}$. If $\left\langle R_{0}\right\rangle(I)$ denotes the mean size of sources with surface brightness $I$, an estimate of the local magnification is $\mu=\left(\langle R\rangle(I) /\left\langle R_{0}\right\rangle(I)\right)^{2}$, where $\langle R\rangle(I)$ is the local average of image sizes with surface brightness $I^{4}{ }^{4}$

\footnotetext{
${ }^{2}$ The importance of the modifications of the KS method discussed in this and the previous section depends on the data set and the mass distribution. For example, if one has a fairly large CCD field centered on an isolated cluster, the finite-field corrections will be moderate, and except for the central part of the cluster, the resulting mass distribution will only be weakly affected by the non-linear corrections. However, if there is a significant mass component close to the edge, or outside of the data field, or if the data field is small, or if one is interested in the distribution in the central part of the cluster, these corrections no longer provide 'minor modifications', but are essential. The WFPC2 images of cluster centers can be used to construct their mass profile, as demonstrated in the contribution by C. Seitz for the cluster $0939+4713$ (Seitz et al. 1995), and one should not even think about attempting this reconstruction without the two modifications discussed here (of course we did to see the - huge - differences). Note that the aperture densiometry (Kaiser 1995), also discussed in the contribution by J. Miralda-Escudé, yields unbiased lower limits of the mass inside circular apertures.

${ }^{3}$ Whereas the faint blue galaxies have $\alpha \approx 1$ and are thus unusable for this effect, the red galaxies appear to have flatter counts, $\alpha \sim 0.4$, so that magnification depletes the number counts locally. In fact, this depletion has been seen in the inner part of A1689 (Broadhurst 1995) and in Cl0939 (Seitz et al. 1995).

${ }^{4}$ The intrinsic size $\left\langle R_{0}\right\rangle(I)$ can be obtained from images in empty fields. The accuracy of the local determination of $\mu$ depends of course on the intrinsic width of the size distribution at fixed surface brightness; in the galaxy model used in Bartelmann \& Narayan (1995), this comes out to be $\Delta \ln R \sim 0.5$. With such a distribution, the size effect alone can provide a reasonable estimate of the surface mass distribution. But even if the width of the intrinsic size distribution turns out to be significantly broader, the size effect averaged over the data field allows breaking of the degeneracy.
} 


\section{Conclusions}

Unbiased, non-linear cluster reconstruction methods, generalizing the original KS method, have been developed; they are quantitatively tested on synthetic data, are easy to apply, and therefore should be applied! In cases where the center of a cluster should be reconstructed from HST images, the modifications discussed here are essential. Currently we (with S. Seitz, M. Bartelmann \& R. Narayan) are developing an improved inversion technique which (1) avoids the introduction of an arbitrary smoothing length needed for performing the local averages, (2) accounts for both the distortion and the magnification effects in a single step, and (3) yields an objective measure for the quality of the reconstruction. The method maximizes the likelihood for the observed image ellipticities and sizes (and/or local number densities) with respect to the surface mass density described on a grid. In order to avoid overfitting the data, the likelihood function is regularized. See the contribution by S. Seitz for some details. Whereas this method is significantly more complicated than the direct inversion (1) or (5), the effort is still negligible compared to obtaining the data, and it most likely will increase the accuracy of the inversion substantially.

Acknowledgements: I thank C. and S. Seitz for helpful comments on this manuscript. This work was supported by the "Sonderforschungsbereich 375-95 für Astro-Teilchenphysik" der Deutschen Forschungsgemeinschaft.

\section{References}

Bartelmann, M., 1995, A\&A, in press

Bartelmann, M. \& Narayan, R., 1995, ApJ, in press

Bonnet, H., Mellier, Y. \& Fort, B., 1994, ApJL, 427, L83

Broadhurst, T.J., 1995, preprint

Broadhurst, T.J., Taylor, A.N. \& Peacock, J.A., 1995, ApJ, 438, 49

Fahlman, G., Kaiser, N., Squires, G. \& Woods, D., 1994, ApJ, 437, 56

Fort, B. \& Mellier, Y., 1994, A\&AR, 5, 239

Fort, B., Prieur, J.L., Mathez, G., Mellier, Y. \& Soucail, G., 1988, A\&A, 200, L17

Gorenstein, M.V., Falco, E.E. \& Shapiro, I.I., 1988, ApJ, 327, 693

Kaiser, N., 1995, ApJL, 439, L1

Kaiser, N. \& Squires, G., 1993, ApJ, 404, 441

Kaiser, N., Squires, G., Fahlman, G., Woods, D.\& Broadhurst, T., 1995, preprint

Kochanek, C.S., 1990, MNRAS, 247, 135

Miralda-Escude, J., 1991, ApJ, 370, 1

Schneider, P., 1995, A\&A, in press

Schneider, P. \& Seitz, C., 1995, A\&A, 294, 411

Seitz, C. \& Schneider, P., 1995a, A\&A, 297, 287

Seitz, S. \& Schneider, P., 1995b, A\&A, in press

Seitz, C. \& Schneider, P., 1995c, in preparation

Seitz, C., Kneib, J.-P., Schneider, P. \& Seitz, S., 1995, in preparation

Smail, I., Ellis, R.S., Fitchett, M.J. \& Edge, A.C., 1995, MNRAS, 273, 277

Tyson, J.A., Valdes, F. \& Wenk, R.A., 1990, ApJ, 349, L1 